\title{
A Preliminary Distance to the Small Magellanic Cloud by the Surface Brightness Technique
}

\author{
Thomas G. Barnes III \\ McDonald Observatory \\ The University of Texas at Austin \\ Thomas J. Moffett \\ Department of Physics \\ Purdue University \\ and \\ Wolfgang P. Gieren \\ Astrophysics Group \\ Physics Faculty \\ Pontificia Universidad Católica de Chile
}

\begin{abstract}
We present a new distance determination to the Small Magellanic Cloud from the surface brightness technique applied to the Cepheid variable HV 829. Although this is a preliminary distance based on only one star, it illustrates the power of the surface brightness technique to extragalactic Cepheid distances, it develops the technique which we will apply to additional SMC and LMC Cepheids, and the distance is of intrinsic interest because of the current controvery concerning distances for the Magellanic Clouds.

For HV 829 itself we obtain a distance modulus of $18.91 \pm 0.20 \mathrm{mag}$. From other evidence we infer that HV 829 is slightly in front of the SMC centroid distance. Correcting to the SMC centroid yields a distance to the SMC of $19.05 \pm 0.20$ mag. We stress that this distance modulus is fully independent of any other distance modulus for the SMC, including those based upon Cepheids. Even so, our result agrees more closely with other, independent Cepheid distances than with RR Lyrae distances and main sequence fitting distances.
\end{abstract}

Full details are being published in the Astrophysical Journal. 